\title{
Progress Toward Automatic Segmentation of Soft X-ray Tomograms Using Convolutional Neural Networks
}

Tia Plautz ${ }^{1,2}$, Rosanne Boudreau ${ }^{1,2}$, Jian-Hua Chen ${ }^{1,2}$, Axel Ekman ${ }^{1,2}$, Mark LeGros ${ }^{1,2}$, Gerry McDermott $^{1,2}$, Carolyn Larabel1 ${ }^{1,2}$

1. Department of Anatomy, University of California, San Francisco, San Francisco, CA, USA

2. Molecular Biophysics and Integrated Bioimaging, Lawrence Berkeley Lab, Berkeley, CA, USA

The purpose of this research is to evaluate the effectiveness of a fully-automatic algorithm based on convolutional neural networks for segmentation of soft x-ray tomograms of budding yeast cells.

Image segmentation refers to the partitioning of an image into non-overlapping regions that are homogeneous with respect to a certain characteristic. In this way, segmentation simplifies the representation of the data. In the context of soft x-ray tomography (SXT) of cells, the purpose of segmentation is to identify the boundaries of organelles, and other structures of interest.

At present, image segmentation is a major bottleneck in the data analysis pipeline. In our current workflow, segmentation is a primarily manual task, which is tedious and time consuming. Automatic segmentation is a difficult task because the relatively low contrast to noise ratio makes conventional, unsupervised techniques ineffective, and the limited availability of already-labeled data makes it difficult to use supervised techniques.

SXT is an imaging technique that may be applied to a variety of biological cells. Because it utilizes the intrinsic contrast due to x-ray attenuation, SXT is capable of imaging whole cells in 3D, in a hydrated, near-native state, without the use of stains. As such, this modality is particularly well-suited for visualizing cellular ultrastructure in an unperturbed cellular environment.

The recent and rapid increase in the popularity of artificial neural networks (ANN) and deep learning provides a viable candidate for fully automatic image segmentation. Although the concept of an artificial neural network has existed for several decades [1], they have previously been of limited use due to a lack of computing power. But, recent advances in computing have turned them into practical approaches.

Deep learning is an improvement on classical ANNs that incorporates a greater number of hidden layers in order to extract complicated features and promote learning higher levels of abstraction. MIT technology review, 2013, named deep learning one of the top 10 breakthroughs of 2013 [2]. Since then, deep learning has been applied successfully to a wide variety of tasks. Applications of deep learning in medical imaging [3-6] are particularly significant to our research. Using the foundations developed in these studies, we are designing a convolutional neural network $(\mathrm{CNN})$ for the segmentation of yeast cells.

We have selected yeast cells for this segmentation project. Despite their relatively regular global structure, there is still vast diversity among cells even of the same cell type, such as due to variation in the stage of the cell cycle (Fig. 1), or the presence of mutations. Such diversity increases the difficulty of the segmentation problem. 
Our network architecture is based on that of Moeskops et al. [4] who uses a multi-scale approach in order to combine the advantages of small convolutional kernels, such as robustness to overfitting, with those of larger kernels, which implicitly provide spatial information. Moeskops et al. also uses a triplanar ("2.5D") structural element, which improves the performance of the classifier by providing information from orthogonal views without excessive computational requirements.

We would like to be able to segment the organelles of any type of cell, which may have a very different global architecture from that of yeast. Because they learn abstract features, neural networks are highly adaptable; features learned from one data set may be transferred to another. This is yet another advantage of using a neural network. Studies of "feature transfer" and "fine-tuning" [7] suggest that given a pre-trained neural network, new specificity may be learned by the network by using "finetuning" of only the last few layers. This is a promising result, since we may study the fine-tuning of our yeast network to other cell types, with very limited data sets, and without the need for excessive manual labor [8].

\section{References:}

[1] Schmidhuber, Journal of Neural Networks 61 (2015), p. 85.

[2] MIT Technology Review (2013). Available:

https://www.technologyreview.com/lists/technologies/2013/

[3] H.R. Roth et al, IEEE Transactions on Medical Imaging 35.5 (2016), p. 1170.

[4] P. Moeskops et al, IEEE Transactions on Medical Imaging 35.5 (2016), p. 1252.

[5] S. Pereira et al, IEEE Transactions on Medical Imaging 35.5 (2016), p. 1240.

[6] A Prasoon et al, International Conference on Medical Image Computing and Computer-assisted Intervention (2013) p. 246.

[7] N. Tajbakhsh et al, IEEE Transactions on Medical Imaging 35.5 (2016), p. 1299.

[8] The National Center for X-ray Tomography is supported by NIH (P41GM103445) and DOE's Office of Biological and Environmental Research (DE-AC02-5CH11231).

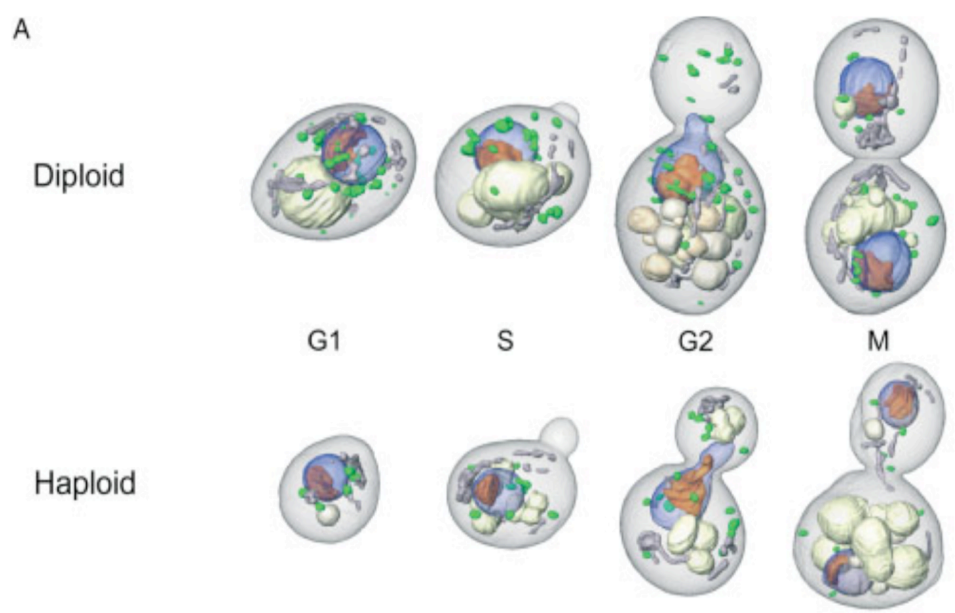

Figure 2. Even among the same cell line there is vast variation in cellular ultrastructure. This complicates the problem of automatic segmentation, rendering conventional unsupervised techniques ineffective. 